\title{
The Rotation Group
}

\author{
Karol Pąk \\ Institute of Informatics \\ University of Białystok \\ Poland
}

\begin{abstract}
Summary. We introduce length-preserving linear transformations of Euclidean topological spaces. We also introduce rotation which preserves orientation (proper rotation) and reverses orientation (improper rotation). We show that every rotation that preserves orientation can be represented as a composition of base proper rotations. And finally, we show that every rotation that reverses orientation can be represented as a composition of proper rotations and one improper rotation.
\end{abstract}

MML identifier: MATRTOP3, version: $\underline{7.12 .014 .167 .1133}$

The papers [11], [35], [36], [8], [10], [9], [3], [7], [14], [2], [30], [4], [19], [12], [31], [24], [34], [13], [22], [17], [1], [20], [15], [16], [40], [38], [33], [25], [28], [37], [23], [6], [39], [18], [21], [32], [5], [26], [29], and [27] provide the terminology and notation for this paper.

\section{Preliminaries}

We adopt the following rules: $x, X$ are sets, $\alpha, \alpha_{1}, \alpha_{2}, r, s$ are real numbers, and $i, j, k, m, n$ are natural numbers.

We now state three propositions:

(1) Let $K$ be a field, $M$ be a square matrix over $K$ of dimension $n$, and $P$ be a permutation of $\operatorname{Seg} n$. Then $\operatorname{Det}\left(\left((M \cdot P)^{\mathrm{T}} \cdot P\right)^{\mathrm{T}}\right)=\operatorname{Det} M$ and for all $i$, $j$ such that $\langle i, j\rangle \in$ the indices of $M$ holds $\left((M \cdot P)^{\mathrm{T}} \cdot P\right)_{i, j}^{\mathrm{T}}=M_{P(i), P(j)}$.

(2) For every field $K$ and for every diagonal square matrix $M$ over $K$ of dimension $n$ holds $M^{\mathrm{T}}=M$.

(C) 2012 University of Białystok CC-BY-SA License ver. 3.0 or late ISSN $1426-2630(p), 1898-9934(e)$ 
(3) For every real-valued finite sequence $f$ and for every $i$ such that $i \in \operatorname{dom} f$ holds $\sum^{2}(f+\cdot(i, r))=\left(\sum^{2} f-f(i)^{2}\right)+r^{2}$.

Let us consider $X$ and let $F$ be a function yielding function. We say that $F$ is $X$-support-yielding if and only if:

(Def. 1) For every function $f$ and for every $x$ such that $f \in \operatorname{dom} F$ and $F(f)(x) \neq$ $f(x)$ holds $x \in X$.

Let us consider $X$. One can check that there exists a function yielding function which is $X$-support-yielding.

Let us consider $X$ and let $Y$ be a subset of $X$. One can check that every function yielding function which is $Y$-support-yielding is also $X$-support-yielding.

Let $X, Y$ be sets. Note that every function yielding function which is $X$ support-yielding and $Y$-support-yielding is also $X \cap Y$-support-yielding. Let $f$ be an $X$-support-yielding function yielding function and let $g$ be a $Y$-supportyielding function yielding function. Note that $f \cdot g$ is $X \cup Y$-support-yielding.

Let us consider $n$. Observe that there exists a function from $\mathcal{E}_{\mathrm{T}}^{n}$ into $\mathcal{E}_{\mathrm{T}}^{n}$ which is homogeneous.

Let us consider $n, m$. Observe that every function from $\mathcal{E}_{\mathrm{T}}^{n}$ into $\mathcal{E}_{\mathrm{T}}^{m}$ is finite sequence-yielding.

Let us consider $n, m$ and let $A$ be a matrix over $\mathbb{R}_{\mathrm{F}}$ of dimension $n \times m$. One can check that Mx2Tran $A$ is additive.

Let us consider $n$ and let $A$ be a square matrix over $\mathbb{R}_{\mathrm{F}}$ of dimension $n$. Note that Mx2Tran $A$ is homogeneous.

Let us consider $n$ and let $f, g$ be homogeneous functions from $\mathcal{E}_{\mathrm{T}}^{n}$ into $\mathcal{E}_{\mathrm{T}}^{n}$. Note that $f \cdot g$ is homogeneous.

\section{IMPROPER ROTATION}

In the sequel $p, q$ are points of $\mathcal{E}_{\mathrm{T}}^{n}$.

Let us consider $n, i$. Let us assume that $i \in \operatorname{Seg} n$. The axial symmetry of $i$ and $n$ yields an invertible square matrix over $\mathbb{R}_{F}$ of dimension $n$ and is defined by the conditions (Def. 2).

(Def. 2)(i) (The axial symmetry of $i$ and $n)_{i, i}=-1_{\mathbb{R}_{\mathrm{F}}}$, and

(ii) for all $k, m$ such that $\langle k, m\rangle \in$ the indices of the axial symmetry of $i$ and $n$ holds if $k=m$ and $k \neq i$, then (the axial symmetry of $i$ and $n)_{k, k}=1_{\mathbb{R}_{\mathrm{F}}}$ and if $k \neq m$, then (the axial symmetry of $i$ and $\left.n\right)_{k, m}=0_{\mathbb{R}_{\mathrm{F}}}$.

The following propositions are true:

(4) If $i \in \operatorname{Seg} n$, then Det (the axial symmetry of $i$ and $n)=-1_{\mathbb{R}_{\mathrm{F}}}$.

(5) If $i, j \in \operatorname{Seg} n$ and $i \neq j$, then $\left({ }^{@} p\right)$ ) (the axial symmetry of $i$ and $n)_{\square, j}=p(j)$.

(6) If $i \in \operatorname{Seg} n$, then $\left({ }^{@} p\right) \cdot(\text { the axial symmetry of } i \text { and } n)_{\square, i}=-p(i)$. 
(7) Suppose $i \in \operatorname{Seg} n$. Then

(i) the axial symmetry of $i$ and $n$ is diagonal, and

(ii) (the axial symmetry of $i$ and $n)^{\smile}=$ the axial symmetry of $i$ and $n$.

(8) If $i \in \operatorname{Seg} n$ and $i \neq j$, then (Mx2Tran (the axial symmetry of $i$ and $n))(p)(j)=p(j)$.

(9) If $i \in \operatorname{Seg} n$, then $(\operatorname{Mx} 2 \operatorname{Tran}($ the axial symmetry of $i$ and $n))(p)(i)=$ $-p(i)$.

(10) If $i \in \operatorname{Seg} n$, then (Mx2Tran (the axial symmetry of $i$ and $n))(p)=$ $p+\cdot(i,-p(i))$.

(11) If $i \in \operatorname{Seg} n$, then Mx2Tran (the axial symmetry of $i$ and $n$ ) is $\{i\}$ support-yielding.

(12) For all elements $a, b$ of $\mathbb{R}_{\mathrm{F}}$ such that $a=\cos r$ and $b=\sin r$ holds Det (the $0_{\mathbb{R}_{\mathrm{F}}}$-block diagonal of $\left.\left\langle\left(\begin{array}{cc}a & b \\ -b & a\end{array}\right), I_{\mathbb{R}_{\mathrm{F}}}^{n \times n}\right\rangle\right)=1_{\mathbb{R}_{\mathrm{F}}}$.

\section{Proper Rotation}

Let us consider $n, \alpha$ and let us consider $i, j$. Let us assume that $1 \leq i<j \leq$ $n$. The functor Rotation $(i, j, n, \alpha)$ yielding an invertible square matrix over $\mathbb{R}_{\mathrm{F}}$ of dimension $n$ is defined by the conditions (Def. 3 ).

(Def. 3)(i) (Rotation $(i, j, n, \alpha))_{i, i}=\cos \alpha$,

(ii) $(\operatorname{Rotation}(i, j, n, \alpha))_{j, j}=\cos \alpha$,

(iii) $(\operatorname{Rotation}(i, j, n, \alpha))_{i, j}=\sin \alpha$,

(iv) $\quad(\operatorname{Rotation}(i, j, n, \alpha))_{j, i}=-\sin \alpha$, and

(v) for all $k, m$ such that $\langle k, m\rangle \in$ the indices of $\operatorname{Rotation}(i, j, n, \alpha)$ holds if $k=m$ and $k \neq i$ and $k \neq j$, then $(\operatorname{Rotation}(i, j, n, \alpha))_{k, k}=1_{\mathbb{R}_{\mathrm{F}}}$ and if $k \neq m$ and $\{k, m\} \neq\{i, j\}$, then $(\operatorname{Rotation}(i, j, n, \alpha))_{k, m}=0_{\mathbb{R}_{\mathrm{F}}}$.

We now state a number of propositions:

(13) If $1 \leq i<j \leq n$, then $\operatorname{Det} \operatorname{Rotation}(i, j, n, \alpha)=1_{\mathbb{R}_{\mathrm{F}}}$.

(14) If $1 \leq i<j \leq n$ and $k \in \operatorname{Seg} n$ and $k \neq i$ and $k \neq j$, then ( $\left({ }^{@} p\right)$. $(\operatorname{Rotation}(i, j, n, \alpha))_{\square, k}=p(k)$.

(15) If $1 \leq i<j \leq n$, then $\left({ }^{@} p\right) \cdot(\operatorname{Rotation}(i, j, n, \alpha))_{\square, i}=p(i) \cdot \cos \alpha+p(j) \cdot$ $-\sin \alpha$.

(16) If $1 \leq i<j \leq n$, then $\left({ }^{@} p\right) \cdot(\operatorname{Rotation}(i, j, n, \alpha))_{\square, j}=p(i) \cdot \sin \alpha+p(j) \cdot$ $\cos \alpha$.

(17) If $1 \leq i<j \leq n$, then $\operatorname{Rotation}\left(i, j, n, \alpha_{1}\right) \cdot \operatorname{Rotation}\left(i, j, n, \alpha_{2}\right)=$ $\operatorname{Rotation}\left(i, j, n, \alpha_{1}+\alpha_{2}\right)$.

(18) If $1 \leq i<j \leq n$, then $\operatorname{Rotation}(i, j, n, 0)=I_{\mathbb{R}_{\mathrm{F}}}^{n \times n}$.

(19) If $1 \leq i<j \leq n$, then $\operatorname{Rotation}(i, j, n, \alpha)$ is orthogonal and $(\operatorname{Rotation}(i, j, n, \alpha))^{\smile}=\operatorname{Rotation}(i, j, n,-\alpha)$. 
(20) If $1 \leq i<j \leq n$ and $k \neq i$ and $k \neq j$, then $(\mathrm{Mx} 2 \operatorname{Tran} \operatorname{Rotation}(i, j, n, \alpha))(p)(k)=p(k)$.

(21) If $1 \leq i<j \leq n$, then $(\mathrm{Mx} 2 \operatorname{Tran} \operatorname{Rotation}(i, j, n, \alpha))(p)(i)=p(i) \cdot \cos \alpha+$ $p(j) \cdot-\sin \alpha$.

(22) $\quad$ If $1 \leq i<j \leq n$, then $(\operatorname{Mx} 2 \operatorname{Tran} \operatorname{Rotation}(i, j, n, \alpha))(p)(j)=p(i) \cdot \sin \alpha+$ $p(j) \cdot \cos \alpha$.

(23) If $1 \leq i<j \leq n$, then $(\operatorname{Mx} 2 \operatorname{Tran} \operatorname{Rotation}(i, j, n, \alpha))(p)=\left(p\left\lceil\left(i-{ }^{\prime} 1\right)\right)^{\frown}\right.$ $\langle p(i) \cdot \cos \alpha+p(j) \cdot-\sin \alpha\rangle^{\frown}\left(p_{l i} \uparrow\left(j-^{\prime} i-^{\prime} 1\right)\right)^{\frown}\langle p(i) \cdot \sin \alpha+p(j) \cdot \cos \alpha\rangle^{\frown}\left(p_{l j}\right)$.

(24) If $1 \leq i<j \leq n$ and $s^{2} \leq p(i)^{2}+p(j)^{2}$, then there exists $\alpha$ such that $(\mathrm{Mx} 2 \operatorname{Tran} \operatorname{Rotation}(i, j, n, \alpha))(p)(i)=s$.

(25) If $1 \leq i<j \leq n$ and $s^{2} \leq p(i)^{2}+p(j)^{2}$, then there exists $\alpha$ such that $(\mathrm{Mx} 2 \operatorname{Tran} \operatorname{Rotation}(i, j, n, \alpha))(p)(j)=s$.

(26) If $1 \leq i<j \leq n$, then $\mathrm{Mx} 2 \operatorname{Tran} \operatorname{Rotation}(i, j, n, \alpha)$ is $\{i, j\}$-supportyielding.

\section{Length-Preserving Linear Transformations}

Let us consider $n$ and let $f$ be a function from $\mathcal{E}_{\mathrm{T}}^{n}$ into $\mathcal{E}_{\mathrm{T}}^{n}$. We say that $f$ is rotation if and only if:

(Def. 4) $\quad|p|=|f(p)|$.

One can prove the following proposition

(27) If $i \in \operatorname{Seg} n$, then Mx2Tran (the axial symmetry of $i$ and $n$ ) is rotation.

Let us consider $n$ and let $f$ be a function from $\mathcal{E}_{\mathrm{T}}^{n}$ into $\mathcal{E}_{\mathrm{T}}^{n}$. We say that $f$ is base rotation if and only if the condition (Def. 5) is satisfied.

(Def. 5) There exists a finite sequence $F$ of elements of the semigroup of functions onto the carrier of $\mathcal{E}_{\mathrm{T}}^{n}$ such that $f=\prod F$ and for every $k$ such that $k \in \operatorname{dom} F$ there exist $i, j, r$ such that $1 \leq i<j \leq n$ and $F(k)=$ Mx2Tran $\operatorname{Rotation}(i, j, n, r)$.

Let us consider $n$. One can check that $\operatorname{id}_{\mathcal{E}_{\mathrm{T}}^{n}}$ is base rotation.

Let us consider $n$. One can check that there exists a function from $\mathcal{E}_{\mathrm{T}}^{n}$ into $\mathcal{E}_{\mathrm{T}}^{n}$ which is base rotation.

Let us consider $n$ and let $f, g$ be base rotation functions from $\mathcal{E}_{\mathrm{T}}^{n}$ into $\mathcal{E}_{\mathrm{T}}^{n}$. One can check that $f \cdot g$ is base rotation.

Next we state the proposition

(28) If $1 \leq i<j \leq n$, then $\mathrm{Mx} 2 \operatorname{Tran} \operatorname{Rotation}(i, j, n, r)$ is base rotation.

Let us consider $n$. Observe that every function from $\mathcal{E}_{\mathrm{T}}^{n}$ into $\mathcal{E}_{\mathrm{T}}^{n}$ which is base rotation is also homogeneous, additive, rotation, and homeomorphism.

Let us consider $n$ and let $f$ be a base rotation function from $\mathcal{E}_{\mathrm{T}}^{n}$ into $\mathcal{E}_{\mathrm{T}}^{n}$. Note that $f^{-1}$ is base rotation. 
Let us consider $n$ and let $f, g$ be rotation functions from $\mathcal{E}_{\mathrm{T}}^{n}$ into $\mathcal{E}_{\mathrm{T}}^{n}$. One can check that $f \cdot g$ is rotation.

In the sequel $f, f_{1}, f_{2}$ are homogeneous additive functions from $\mathcal{E}_{\mathrm{T}}^{n}$ into $\mathcal{E}_{\mathrm{T}}^{n}$.

Let us consider $n$ and let us consider $f$. The functor AutMt $f$ yields a square matrix over $\mathbb{R}_{\mathrm{F}}$ of dimension $n$ and is defined as follows:

(Def. 6) $f=$ Mx2Tran AutMt $f$.

Next we state several propositions:

(29) $\operatorname{AutMt}\left(f_{1} \cdot f_{2}\right)=\operatorname{AutMt} f_{2} \cdot \operatorname{AutMt} f_{1}$.

(30) Suppose $k \in X$ and $k \in \operatorname{Seg} n$. Then there exists $f$ such that

(i) $f$ is $X$-support-yielding and base rotation,

(ii) if $\overline{\overline{X \cap \operatorname{Seg} n}}>1$, then $f(p)(k) \geq 0$, and

(iii) for every $i$ such that $i \in X \cap \operatorname{Seg} n$ and $i \neq k$ holds $f(p)(i)=0$.

(31) For every subset $A$ of $\mathcal{E}_{\mathrm{T}}^{n}$ such that $f\left\lceil A=\operatorname{id}_{A}\right.$ holds $f\left\lceil\operatorname{Lin}(A)=\operatorname{id}_{\operatorname{Lin}(A)}\right.$.

(32) Let $A$ be a subset of $\mathcal{E}_{\mathrm{T}}^{n}$. Suppose $f$ is rotation and $f\left\lceil A=\mathrm{id}_{A}\right.$. Let given $i$. Suppose $i \in \operatorname{Seg} n$ and the base finite sequence of $n$ and $i \in \operatorname{Lin}(A)$. Then $f(p)(i)=p(i)$.

(33) Let $f$ be a rotation function from $\mathcal{E}_{\mathrm{T}}^{n}$ into $\mathcal{E}_{\mathrm{T}}^{n}$. Suppose $f$ is $X$-supportyielding and for every $i$ such that $i \in X \cap \operatorname{Seg} n$ holds $p(i)=0$. Then $f(p)=p$.

(34) If $i \in \operatorname{Seg} n$ and $n \geq 2$, then there exists $f$ such that $f$ is base rotation and $f(p)=p+\cdot(i,-p(i))$.

(35) If $f$ is $\{i\}$-support-yielding and rotation, then AutMt $f=$ the axial symmetry of $i$ and $n$ or AutMt $f=I_{\mathbb{R}_{\mathrm{F}}}^{n \times n}$.

(36) If $f_{1}$ is rotation, then there exists $f_{2}$ such that $f_{2}$ is base rotation and $f_{2} \cdot f_{1}$ is $\{n\}$-support-yielding.

\section{Rotation Matrix Classification}

The following three propositions are true:

(37) If $f$ is rotation, then Det AutMt $f=1_{\mathbb{R}_{\mathrm{F}}}$ iff $f$ is base rotation.

(38) If $f$ is rotation, then Det AutMt $f=1_{\mathbb{R}_{\mathrm{F}}}$ or Det AutMt $f=-1_{\mathbb{R}_{\mathrm{F}}}$.

(39) If $f_{1}$ is rotation and Det AutMt $f_{1}=-1_{\mathbb{R}_{\mathrm{F}}}$ and $i \in \operatorname{Seg} n$ and AutMt $f_{2}=$ the axial symmetry of $i$ and $n$, then $f_{1} \cdot f_{2}$ is base rotation.

Let us consider $n$ and let $f$ be a rotation homogeneous additive function from $\mathcal{E}_{\mathrm{T}}^{n}$ into $\mathcal{E}_{\mathrm{T}}^{n}$. One can check that AutMt $f$ is orthogonal.

Let us consider $n$. One can verify that every function from $\mathcal{E}_{\mathrm{T}}^{n}$ into $\mathcal{E}_{\mathrm{T}}^{n}$ which is homogeneous, additive, and rotation is also homeomorphism. 


\section{The Rotation Mapping a Given Point to Another Point}

One can prove the following propositions:

(40) Suppose $n=1$ and $|p|=|q|$. Then there exists $f$ such that $f$ is rotation and $f(p)=q$ either AutMt $f=$ the axial symmetry of $n$ and $n$ or AutMt $f=I_{\mathbb{R}_{\mathrm{F}}}^{n \times n}$.

(41) If $n \neq 1$ and $|p|=|q|$, then there exists $f$ such that $f$ is base rotation and $f(p)=q$.

\section{REFERENCES}

[1] Grzegorz Bancerek. Cardinal numbers. Formalized Mathematics, 1(2):377-382, 1990.

[2] Grzegorz Bancerek. The fundamental properties of natural numbers. Formalized Mathematics, 1(1):41-46, 1990.

[3] Grzegorz Bancerek. The ordinal numbers. Formalized Mathematics, 1(1):91-96, 1990.

[4] Grzegorz Bancerek and Krzysztof Hryniewiecki. Segments of natural numbers and finite sequences. Formalized Mathematics, 1(1):107-114, 1990.

[5] Grzegorz Bancerek and Andrzej Trybulec. Miscellaneous facts about functions. Formalized Mathematics, 5(4):485-492, 1996.

[6] Czesław Byliński. Binary operations. Formalized Mathematics, 1(1):175-180, 1990.

[7] Czesław Byliński. The complex numbers. Formalized Mathematics, 1(3):507-513, 1990.

[8] Czesław Byliński. Functions and their basic properties. Formalized Mathematics, 1(1):5565, 1990.

[9] Czesław Byliński. Functions from a set to a set. Formalized Mathematics, 1(1):153-164, 1990.

[10] Czesław Byliński. Partial functions. Formalized Mathematics, 1(2):357-367, 1990.

[11] Czesław Byliński. Some basic properties of sets. Formalized Mathematics, 1(1):47-53, 1990.

[12] Czesław Byliński. The sum and product of finite sequences of real numbers. Formalized Mathematics, 1(4):661-668, 1990.

[13] Agata Darmochwał. The Euclidean space. Formalized Mathematics, 2(4):599-603, 1991.

[14] Krzysztof Hryniewiecki. Basic properties of real numbers. Formalized Mathematics, 1(1):35-40, 1990.

[15] Katarzyna Jankowska. Matrices. Abelian group of matrices. Formalized Mathematics, 2(4):475-480, 1991.

[16] Katarzyna Jankowska. Transpose matrices and groups of permutations. Formalized Mathematics, 2(5):711-717, 1991.

[17] Artur Korniłowicz and Yasunari Shidama. Intersections of intervals and balls in $\mathcal{E}_{\mathrm{T}}^{n}$. Formalized Mathematics, 12(3):301-306, 2004.

[18] Jarosław Kotowicz. Real sequences and basic operations on them. Formalized Mathematics, 1(2):269-272, 1990.

[19] Jarosław Kotowicz. Functions and finite sequences of real numbers. Formalized Mathematics, 3(2):275-278, 1992.

[20] Eugeniusz Kusak, Wojciech Leończuk, and Michał Muzalewski. Abelian groups, fields and vector spaces. Formalized Mathematics, 1(2):335-342, 1990.

[21] Anna Lango and Grzegorz Bancerek. Product of families of groups and vector spaces. Formalized Mathematics, 3(2):235-240, 1992.

[22] Michał Muzalewski. Categories of groups. Formalized Mathematics, 2(4):563-571, 1991.

[23] Yatsuka Nakamura. Determinant of some matrices of field elements. Formalized Mathematics, 14(1):1-5, 2006, doi:10.2478/v10037-006-0001-4.

[24] Beata Padlewska and Agata Darmochwał. Topological spaces and continuous functions. Formalized Mathematics, 1(1):223-230, 1990.

[25] Karol Pąk. Basic properties of determinants of square matrices over a field. Formalized Mathematics, 15(1):17-25, 2007, doi:10.2478/v10037-007-0003-x. 
[26] Karol Pąk. Basic properties of the rank of matrices over a field. Formalized Mathematics, 15(4):199-211, 2007, doi:10.2478/v10037-007-0024-5.

[27] Karol Pąk. Block diagonal matrices. Formalized Mathematics, 16(3):259-267, 2008, doi:10.2478/v10037-008-0031-1.

[28] Karol Pąk. Linear transformations of Euclidean topological spaces. Formalized Mathematics, 19(2):103-108, 2011, doi: 10.2478/v10037-011-0016-3.

[29] Nobuyuki Tamura and Yatsuka Nakamura. Determinant and inverse of matrices of real elements. Formalized Mathematics, 15(3):127-136, 2007, doi:10.2478/v10037-007-0014-7.

[30] Andrzej Trybulec. Binary operations applied to functions. Formalized Mathematics, 1(2):329-334, 1990.

[31] Andrzej Trybulec and Czesław Byliński. Some properties of real numbers. Formalized Mathematics, 1(3):445-449, 1990.

[32] Wojciech A. Trybulec. Basis of real linear space. Formalized Mathematics, 1(5):847-850, 1990.

[33] Wojciech A. Trybulec. Groups. Formalized Mathematics, 1(5):821-827, 1990.

[34] Wojciech A. Trybulec. Vectors in real linear space. Formalized Mathematics, 1(2):291-296, 1990.

[35] Zinaida Trybulec. Properties of subsets. Formalized Mathematics, 1(1):67-71, 1990.

[36] Edmund Woronowicz. Relations and their basic properties. Formalized Mathematics, 1(1):73-83, 1990.

[37] Yuguang Yang and Yasunari Shidama. Trigonometric functions and existence of circle ratio. Formalized Mathematics, 7(2):255-263, 1998

[38] Xiaopeng Yue, Xiquan Liang, and Zhongpin Sun. Some properties of some special matrices. Formalized Mathematics, 13(4):541-547, 2005.

[39] Katarzyna Zawadzka. The sum and product of finite sequences of elements of a field. Formalized Mathematics, 3(2):205-211, 1992.

[40] Katarzyna Zawadzka. The product and the determinant of matrices with entries in a field. Formalized Mathematics, 4(1):1-8, 1993.

Received May 30, 2011 